\title{
Gallium Maltolate
}

National Cancer Institute

\section{Source}

National Cancer Institute. Gallium Maltolate. NCI Thesaurus. Code C133020.

An orally bioavailable form of the element gallium (Ga) composed of a trivalent gallium cation (Ga3+) coordinated to three maltolate ligands, with anti-inflammatory, antiproliferative, antineoplastic, analgesic, antiresorptive and antibacterial activities. Upon administration of gallium maltolate, Ga3+, which is structurally similar to the ferric ion (Fe3+), competes with and replaces Fe3+ in many vital Fe 3+-mediated biological reactions.. Unlike Fe3+, Ga3+ cannot be reduced, cannot participate in redox reactions and cannot mimic Fe3+ functions. In rapidly proliferating cells, such as cancer cells, high amounts of iron are needed for DNA synthesis. The incorporation of Ga3+ inactivates the Fe3+-dependent enzyme ribonucleotide reductase (RR), an enzyme essential for DNA synthesis, leading to an inhibition of DNA synthesis and induction of cell death in rapidly proliferating cells. Gallium similarly reduces bacterial cell growth. In addition, Ga3+ is able to suppress inflammation through the down-regulation of pro-inflammatory cells and the inhibition of pro-inflammatory cytokine secretion. Gallium also exerts analgesic effects due to the inhibition of Fe3+-dependent enzymes involved in inflammation and may interfere with the activity of certain metalloproteinases and neuropeptides that are implicated in pain. Also, gallium is able to inhibit bone resorption by osteoclasts, may inhibit metastasis to bone and may prevent the destruction of bone by tumors. 